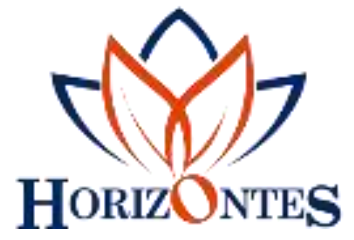

www.revistahorizontes.org

\title{
Importancia de la asignatura cátedra Integradora en el desarrollo de los estudiantes de Profesionalización de la UNAE
}

\author{
Importance of the Integrative chair in the development of UNAE students for \\ professionalization
}

Importância da cátedra Integrativa no desenvolvimento dos alunos da profissionalização $U N A E$

ARTÍCULO DE INVESTIGACIÓN

Karina Maribel Castillo Pinos

karina.castillo@unae.edu.ec

ORCID: 0000-0002-1154-1297

\author{
Juan Alejandro Rodríguez Carrión \\ juanalerd@gmail.com \\ ORCID: 0000-0002-3199-3258
}

\section{Dilida Anayra Luengo Molero \\ dilida.luengo@unae.edu.ec \\ ORCID: 0000-0003-0859-8628}

\section{Universidad Nacional de Educación, Ecuador}

Recibido 10 de noviembre 2020 | Arbitrado y aceptado 20 de enero 2021| Publicado en marzo 2021

\section{RESUMEN}

La asignatura de Cátedra Integradora se ha constituido en una herramienta implementada para ayudar a los estudiantes universitarios a desarrollar investigaciones y proyectos que les permitirá vincularse con su profesión durante el proceso académico de titulación. Por lo tanto, se consideró pertinente establecer desde una perspectiva teórica la importancia de la asignatura de Cátedra Integradora en el desarrollo de los estudiantes de Profesionalización de la UNAE. En este sentido, la limitada disponibilidad de material informativo sobre la relevancia de esta asignatura en la UNAE puso en evidencia la necesidad de llevar a cabo la presente investigación; en cuyo caso, se desarrolló a partir de un estudio de carácter explicativo y descriptivo considerando como población a los estudiantes de la carrera de Profesionalización y docentes de la asignatura para el levantamiento de la información.

Palabras clave: Cátedra integradora; educación; asignatura; profesionalización

\section{ABSTRACT}

The Integrative Chair course has become a tool implemented to help university students develop research and projects that will allow them to link with their profession during the academic degree process. Therefore, it was considered pertinent to establish from a theoretical perspective the importance of the Integrative Chair subject in the development of UNAE Professionalization students. In this sense, the limited availability of informative material on the relevance of this subject at UNAE highlighted the need to carry out this research; in which case, it was developed from an explanatory and descriptive study considering as population the students of the Professionalization career and teachers of the subject for the gathering of information.

Key words: Integrative chair; education; subject; professionalization 


\section{RESUMO}

O curso Cátedra Integrativa tornou-se uma ferramenta implementada para auxiliar os universitários a desenvolver pesquisas e projetos que lhes permitam vincular-se à profissão durante o processo de graduação. Portanto, considerou-se pertinente estabelecer do ponto de vista teórico a importância do curso de Cátedra Integrativa no desenvolvimento dos alunos da Profissionalização da UNAE. Nesse sentido, a limitada disponibilidade de material informativo sobre a relevância do tema na UNAE evidenciou a necessidade de realização da presente investigação; nesse caso, desenvolveuse a partir de um estudo explicativo e descritivo considerando como população os alunos da carreira de profissionalização e professores da disciplina para a coleta de informações.

Palavras-chave: Cadeira Integrativa; Educação; sujeito; profissionalização

\section{INTRODUCCIÓN}

En los últimos años se ha evidenciado un cambio de paradigmas en el sistema educativo de nivel superior, el cual ha sido objeto de una serie de restructuraciones y mejoras orientadas a garantizar la calidad de la enseñanza. En consecuencia es posible identificar que los nuevos modelos educativos implementados no solo buscan promover el aprendizaje teórico-práctico, sino también fomentar un aprendizaje reflexivo y analítico que les permita a los estudiantes desarrollar un sinnúmero de habilidades y capacidades necesarias para desempeñarse satisfactoriamente en sus respectivos campos profesionales a futuro (Narváez-Peña, SalinasJumbo, y Curay-Jumbo, 2019).

Para alcanzar un adecuado desempeño profesional resulta fundamental que los modelos de enseñanza se encuentren alineados a las transformaciones sociales que se experimentan en cada país, a fin de que puedan propiciar mejoras significativas mediante la investigación, la innovación y el desarrollo (Aguilar, Apolo, y Rodríguez, 2018). Es por esto que resulta necesario para los estudiantes tener un acercamiento con su área de trabajo, para ello diversas instituciones de tercer y cuarto nivel han implementado en su currículo la asignatura de Cátedra Integradora con la cual buscan vincular a los estudiantes con su campo profesional mediante investigaciones y el desarrollo de proyectos.

El profesor debe estar
reinventándose como un
investigador y co-creador de
conocimientos que motive la
reflexión y el pensamiento
crítico desde su práctica diaria,
donde el aula sea un espacio de
intervención y la creatividad es
una herramienta pedagógica
que se va creando y en su
creación se revela la creatividad
(Aguilar, Apolo, y Rodríguez,
2018).

En este sentido, uno de los principales propósitos de la asignatura es fomentar un aprendizaje integrador, puesto que a través de la investigación permite vincular conocimientos de diversas áreas que se incluyen en la malla curricular que pueden ser puestos en práctica a través del desarrollo de proyectos. La práctica integradora en la formación de profesionales en el campo pedagógico se ve desde tres direcciones: en primer lugar, se describen los grupos destinatarios de las medidas integradoras, luego se muestra según qué pautas conceptuales de integración se implementan y finalmente se resume el estado actual de la investigación sobre integración de saberes en las instituciones académicas (Díaz-Valdés, Rodríguez-Guerra, \& Valdés-Sáenz, 2017). 
A su vez, resulta pertinente destacar el desafío para los facilitadores de la Cátedra Integradora en el proceso de enseñanza y gestión investigativa, considerando que uno de los requisitos fundamentales radica en la necesidad de contar con una adecuada formación alineada a los nuevos paradigmas educativos en los que la innovación y la integración de saberes se han constituido como pilares fundamentales en la formación de profesionales en el campo pedagógico; así como también una correcta formación didáctica que les permita dirigir la cátedra en función de la amplia variedad de materiales teóricos y alternativas de proyectos investigativos a construir. Por lo tanto, los docentes deben estar preparados para alcanzar un equilibrio entre sus conocimientos previos y los retos que presenta la enseñanza de esta asignatura, a fin de satisfacer las necesidades de los estudiantes que deben aprender a elaborar investigaciones de alto nivel y relevancia científica (Moscoso-Zamora \& Quiñonez-Alvarado, 2018).

La problemática identificada en relación al tema presentado, radica la carencia de estudios que permitan determinar la efectividad de la asignatura de Cátedra integradora para los docentes de la UNAE en su proceso de formación-profesionalización, dado a que si bien se ha demostrado que con un material informativo adecuado, socialización previa de la asignatura, así como la constante práctica en los proyectos investigativos, existen mayores posibilidades de mejorar los niveles de formación profesional de los docentes, aspecto que se buscará indagar a lo largo del presente artículo (Narváez-Peña, Salinas-Jumbo, \& Curay-Jumbo, 2019).
En este contexto, se procede por lo tanto realizar un análisis respecto a la importancia de la asignatura de Cátedra Integradora dentro de la profesionalización que ofrece actualmente la Universidad Nacional de Educación (UNAE) en la provincia de Cañar, direccionada a los docentes estudiantes que ya se encuentran ejerciendo su profesión, pero que sin embargo, tomando en consideración que esta profesión además de demandar personas comprometidas en la enseñanza de los discentes de distintos niveles escolares y asignaturas, requiere de un alto grado de preparación previa y se debe cultivar a su vez de manera constate, con lo cual se garantice que estos maestros podrán cumplir a cabalidad con su rol y lo que demanda en la actualidad el Ministerio de Educación en el Ecuador.

Cabe mencionar que la parte de las actividades que se impulsan en estos docentes en su proceso de preparación, específicamente dentro de la asignatura de Cátedra Integradora son los denominados "Piensas", los cuales se solicitan como propuestas de mini proyectos o artículos sobre temas varios, con lo cual se busca ir familiarizando al maestro tanto sobre la estructura que se demandan para la realización de sus futuros proyectos de pregrado y postgrado, así como para que tengan una noción mucho más amplia tanto de la asignatura, los procesos que deben considerar como parte de sus metodologías de enseñanza, así como para que socialicen a su vez con los alumnos, el desarrollo de trabajos de índole investigativa y práctica.

Como parte del Plan Nacional de Profesionalización estas carreras están dirigidas a docentes en ejercicio con título de bachiller y/o nivel de tecnólogo que no cuenten 
con título de licenciatura en educación, y se encuentren dentro de las provincias del territorio de la Amazonía: Orellana, Sucumbíos, Pastaza, Tena, Morona Santiago y Zamora Chinchipe (UNAE, 2020).

Complementando lo expuesto en el apartado anterior, es posible a su vez detallar que la UNAE constantemente ha destacado en la promoción de la oferta de títulos para docentes que ejercen con mínimo 5 años de experiencia dentro del campo de la docencia en jurisdicciones diversas, abarcando un enfoque intercultural bilingüe para que logren ejercer con mejores garantías los maestros bajo un nombramiento para niveles de educación básica elemental hasta los niveles elementales de educación (Gobernación de Cañar, 2019).

La malla curricular de la UNAE cuenta con una categorización clara, particularmente en la unidad de formación profesional donde se busca, mediante la asignatura de Cátedra Integradora el diseño y desarrollo de procesos de enseñanza personalizada, donde además se destacan el proceso de praxis que se abarcan tanto a nivel teórico y práctico, en estas se destacan temas de inclusión, diversidad, interculturalidad, entre otros tópicos que garanticen el fortalecimiento del sistema educativo desde la educación general básica en las instituciones de educación tanto de la provincia como del país en general.

Con base a lo expuesto previamente, el objetivo general por lo tanto de este estudio será, analizar la importancia de la asignatura de Cátedra Integradora en el desarrollo de los estudiantes de profesionalización de la UNAE.

Para lograr disponer de una mejor concepción de lo que abarca el contexto del presente artículo, se toma lo expuesto por Narváez (2019), "La catedra integradora se constituye en una herramienta para ayudar a los jóvenes universitarios a conocer de cerca cómo va a ser su futuro profesional, porque de forma obligatoria deberán desarrollar diferentes proyectos que los ayudara a vincularse con la sociedad" (párr. 2).

Complementando lo anteriormente expuesto, es posible determinar que, como asignatura, la Cátedra integradora presenta objetivos claros en su aplicación dentro de la malla curricular, dado que abarca procedimientos y una metodología que permite incrementar la tasa de retención de la información y conocimientos que posteriormente les servirán los docentes en su proceso de profesionalización para ser aplicados dentro de las aulas de clases.

\section{MÉTODO}

El estudio se abordó desde el método inductivo-deductivo, considerando que se buscó explicar la importancia de la asignatura de Cátedra Integradora que se imparte a los estudiantes de Profesionalización de la UNAE, siendo este el contexto de partida de la investigación. A su vez, se emplearon estrategias de razonamiento lógico asociadas al pensamiento deductivo, puesto que se complementó la información particular con la revisión teórica sobre el contenido de la cátedra, el enfoque metodológico que permitan destacar la relevancia de la asignatura y derivar en conclusiones generales del caso.

Particularmente, se trabajó con un enfoque de investigación de carácter cualitativa, que de acuerdo a lo que señalaron Baptista, et al. (2014), "se basan más en una lógica y proceso inductivo (explorar y describir, y luego generar perspectivas teóricas). Van de lo particular a lo general" (p. 8).

Consecuentemente, considerando el principal propósito del presente estudio se 
direccionó a establecer la importancia de la asignatura Cátedra Integradora, se consideró pertinente abordar la investigación con base a un enfoque cualitativo, con el cual fue posible examinar el contenido del programa de estudios de la asignatura, así como las percepciones de quienes imparten la asignatura y de los estudiantes, pudiendo establecer conclusiones que permitan la generalización.

En cuanto al alcance del estudio, se trabajó con una modalidad de investigación de tipo explicativo y descriptivo, en primer lugar debido a que se logró identificar a partir de la problemática descrita la limitada disponibilidad de información teórica que permita determinar la importancia de Cátedra Integradora y la necesidad de abordar la asignatura desde un enfoque complementario con otras áreas de conocimiento; así como también se trabajó con una investigación descriptiva puesto que se analizaron las percepciones de los actores involucrados (estudiantes y docentes) con respecto a la asignatura.

A su vez, se trabajó con una modalidad de investigación evaluativa, con la cual fue posible obtener información y evaluar la importancia del programa de estudios de la Cátedra Integradora que se imparte a los estudiantes de Profesionalización de la UNAE; este tipo de estudios resulta especialmente útil en investigaciones educativas, puesto que permite establecer a partir del análisis cualitativo los logros alcanzados en la enseñanza de la asignatura, permitiéndole a la investigadora establecer conclusiones y recomendaciones orientadas a promover la mejora continua en la enseñanza de esta cátedra en pro de la calidad educativa de la institución.

Desde esta perspectiva, el proceso de investigación realizado a partir de las modalidades seleccionadas, se realizó a partir de un proceso sistemático de recopilación de fuentes de información primaria y secundaria, ejecutado técnicamente de acuerdo los siguientes pasos:

- Revisión del programa de estudios de la asignatura Cátedra Integradora que se imparte a los estudiantes de Profesionalización.

- Observación del contexto en que se llevan a cabo los procesos de enseñanza $y$ aprendizaje de la asignatura.

- Fue necesario examinar las perspectivas y opiniones de los involucrados (estudiantes y docentes).

- Contrastar y establecer analogías a partir de los resultados obtenidos de la investigación realizada en la UNAE con la información teórica recopilada a partir de diversos estudios complementarios.

- Establecer conclusiones con las cuales sea posible describir de forma clara la relevancia de la asignatura y establecer posibles recomendaciones que propicien la mejora continua en la enseñanza de la Cátedra Integradora, tanto en la UNAE como en otras instituciones de tercer nivel en las que se imparte dicha asignatura.

La población de estudio considerada para el levantamiento de la información primaria, fueron los estudiantes de Profesionalización de la UNAE que actualmente consta de un total de 2903 (EB 2019 estudiantes y EIB 884 estudiantes) según la información proporcionada por la UNAE (2020), quiénes fueron sometidos al proceso investigativo a través de la aplicación de técnicas e instrumentos de investigación para determinar según su experiencia y perspectiva, cómo se ha abordado la asignatura de Cátedra 
Integradora en su proceso de formación, identificando de esta manera los aspectos importantes sobre la gestión de esta materia en esta institución universitaria que posteriormente fueron contrastados con la información teórica analizada.

En este contexto, considerando el alcance de la investigación y el enfoque seleccionado, se trabajó únicamente con una muestra de diez personas en las que se incluyó a ocho estudiantes de la carrera de Profesionalización de la UNAE y a dos docentes, seleccionados del total de 12 autores que imparten la asignatura de Cátedra Integradora en los diferentes semestres, quienes participaron en un grupo de discusión vía online moderado por la investigadora a fin de conocer sus percepciones respecto a la asignatura.
Esta técnica de investigación resulta especialmente útil para el desarrollo de estudios de carácter social, puesto que ofrece mayor apertura a los participantes de expresar sus diferentes puntos de vista con respecto al tema analizado. En el caso de los ocho estudiantes, la moderadora realizó una serie de preguntas para conocer su percepción sobre la asignatura de Cátedra Integradora; mientras que la participación de los dos docentes seleccionados se direccionó principalmente a ofrecer la retroalimentación pertinente sobre la temática analizada a fin de ampliar las perspectivas de análisis sobre la importancia de la asignatura.

Tabla 1. Pregunta para el grupo de discusión

\begin{tabular}{|c|c|c|}
\hline \multicolumn{3}{|r|}{ Guion de preguntas } \\
\hline № & Clases de preguntas & Preguntas \\
\hline 1 & \multirow{2}{*}{ Preguntas de apertura } & $\begin{array}{l}\text { ¿Cuál es su percepción con respecto a la asignatura de } \\
\text { Cátedra Integradora? }\end{array}$ \\
\hline 2 & & $\begin{array}{l}\text { ¿Considera que la asignatura es relevante para su formación } \\
\text { profesional? }\end{array}$ \\
\hline 3 & \multirow{5}{*}{ Preguntas específicas } & $\begin{array}{l}\text { ¿Considera que el contenido de la asignatura es adecuado a } \\
\text { sus necesidades de aprendizaje? ¿La asignatura cubre sus } \\
\text { expectativas? }\end{array}$ \\
\hline 4 & & $\begin{array}{l}\text { ¿Considera que la formación recibida a través de la Cátedra } \\
\text { Integradora le será especialmente útil para su } \\
\text { desenvolvimiento en el campo laboral? ¿La información } \\
\text { recibida sobre la asignatura le ha sido útil? }\end{array}$ \\
\hline 5 & & $\begin{array}{l}\text { ¿Cuáles considera que han sido las principales aportaciones } \\
\text { de esta asignatura para su formación? }\end{array}$ \\
\hline 6 & & $\begin{array}{l}\text { ¿El programa de estudios de la asignatura lo motiva a ampliar } \\
\text { y profundizar sus conocimientos fuera de clase? }\end{array}$ \\
\hline 7 & & $\begin{array}{l}\text { ¿Considera que se han cumplido o se cumplen los objetivos } \\
\text { establecidos en el programa de estudio de la asignatura? }\end{array}$ \\
\hline
\end{tabular}




\section{Guion de preguntas}

№ $\quad$ Clases de preguntas

8

9

Preguntas de cierre

10
¿Se encuentra satisfecho cursando la asignatura de Cátedra Integradora?

¿Considera importante la asignatura Cátedra Integradora en la formación de los estudiantes de Profesionalización?

$¿$ Considera que existen aspectos que se puedan o deban mejorar en el programa de estudios de la asignatura Cátedra Integradora?
Por otra parte, para la selección de la información documental que se contrastó con los resultados que se obtuvieron a partir de la ejecución del grupo de discusión se consideraron los siguientes criterios de inclusión y exclusión, lo que permitió filtrar la información seleccionando la de mayor relevancia científica y teórica, según su aportación al presente estudio.

- Criterios de inclusión: Estudios desarrollados desde una perspectiva empírica durante los últimos cinco años (artículos científicos, ensayos, informes), estudios realizados en otras instituciones académicas del país o la región en los que se aborde una temática similar.

- Criterios de Exclusión: Publicaciones literarias (libros, editoriales); estudios de mayor antigüedad, estudios en los que se analice la asignatura impartida en otros niveles de educación, estudios publicados en fuentes poco confiables.

\section{RESULTADOS Y DISCUSIÓN}

Los resultados obtenidos del grupo de discusión en el que participaron docentes y estudiantes de la carrera de Profesionalización de la UNAE, proporcionaron información relevante con respecto a la percepción y opiniones de los participantes con respecto a la asignatura de Cátedra Integradora. Siguiendo la guía de preguntas previamente formuladas, en primer lugar se consultó a los panelistas ¿Cuál es su percepción con respecto a la asignatura de Cátedra Integradora?, de lo cual se logró identificar, según las opiniones de los participantes, que la implementación de la asignatura desde un enfoque integral ha favorecido su formación profesional, permitiéndoles asimilar de mejor forma los contenidos de la carrera, así como también desarrollar habilidades para resolución de problemas que suceden en entornos reales.

Por otra parte, con relación a las preguntas realizadas para identificar la relevancia de la asignatura de Cátedra Integradora, los estudiantes y docentes coincidieron en que la implementación de la asignatura ha sido importante para su formación, considerando que les permite plantear escenarios basados en situaciones reales a los cuales deben proponer alternativas de solución; esto según argumentaron, representa una aportación significativa para su desenvolvimiento futuro. Desde esta perspectiva, la asignatura les ofrece la oportunidad de desarrollar proyectos basados en problemas reales del entorno, a fin 
de que puedan plantear alternativas de solución y poner en práctica los conocimientos adquiridos durante su formación profesional.

En este sentido, al realizarles las siguiente interrogante a los estudiantes $i$ Considera que el contenido de la asignatura es adecuado a sus necesidades de aprendizaje?, se obtuvieron distintas opiniones, puesto que en su mayoría los estudiantes consideran adecuados los contenidos de la asignatura, mientras que una minoría de participantes consideran necesario que se incluyan contenidos complementarios que se alineen principalmente con el contexto social y educativo que se experimenta en la actualidad.

Ante estas opiniones, los docentes participantes coincidieron en la importancia de aplicar estrategias curriculares para complementar los contenidos de la Cátedra Integradora y proporcionar una direccionalidad que además de abordar las diferentes asignaturas y unidades de forma vinculativa, sea capaz de alinearse a las realidades contemporáneas de la sociedad; puesto que de lo contrario se corre el riesgo de formar profesionales con falencias en ciertas áreas de conocimiento, lo que a su vez podría traducirse en mayores dificultades para su desenvolvimiento futuro en el campo de la enseñanza.

Sin embargo, agregaron que para lograr el cumplimiento de los objetivos de la asignatura, es necesario que los educadores se encuentren preparados para poder hacer frente a posibles barreras que se presenten durante el proceso de enseñanza, lo que implica mantenerse capacitados y adoptar un rol activo en la actualización conocimientos conforme a las distintas realizadas del entorno social, identificar fuentes válidas de información $\mathrm{y}$ contrastar con enfoques epistemológicos que sustenten las investigaciones y proyectos, a fin de poder diseñar contenidos que se ajusten a los contextos actuales y respondan de mejor manera a las necesidades de aprendizaje de los estudiantes.

Así mismo, enfatizaron la importancia de asumir la enseñanza de Cátedra Integradora de una forma integral, en la que se complementen los elementos fundamentales de la carrera en contraste con las necesidades de promover el desarrollo de conocimientos y habilidades más exactas y específicas con relación a la realidad social y educativa en la que deben desenvolverse los estudiantes de la carrera de Profesionalización.

Por otra parte, para conocer la percepción de los estudiantes sobre la utilidad de la asignatura, se les consultó si: ¿Considera que la formación recibida a través de la Cátedra Integradora le será especialmente útil para su desenvolvimiento en el campo laboral?, y si ¿La información recibida sobre la asignatura le ha sido útil?; de lo cual se obtuvieron respuestas positivas, puesto que en su totalidad los participantes coincidieron en que la asignatura les ha resultado especialmente útil para obtener los conocimientos y aprendizajes necesarios de acuerdo a la pertinencia de su carrera.

Además, en respuesta a la interrogante realizada sobre ¿Cuáles considera que han sido las principales aportaciones de esta asignatura para su formación?, los estudiantes mencionaron que el cursar la asignatura les ha permitido desarrollar saberes y actitudes humanistas; es decir, no solo son capaces de asumir los conocimientos de las diferentes materias que se imparten en la carrera, sino también han logrado analizar su relación con las distintas realidades del entorno local. Esto, según destacaron los participantes, los obliga a desarrollar habilidades de pensamiento crítico y resolución de problemas, puesto que, a 
través de los proyectos e investigaciones, han logrado trasladar dichos conocimientos adquiridos a la búsqueda de posibles soluciones a los problemas identificados en el contexto social y educativo.

A partir de estos argumentos, coincidieron en que la asignatura ha logrado ser una herramienta de motivación para incentivarlos a la búsqueda de nuevos conocimientos y la exploración de distintas realidades $y$ problemas que aún requieren solución. Con base a estas opiniones, los docentes participantes concluyeron que la asignatura sí ha cumplido con los objetivos establecidos en el programa de estudio, a pesar de los desafíos señalados previamente y la necesidad de profundizar en los contenidos alineados a los problemas contemporáneos de la sociedad, principalmente en relación a los problemas que se presentan en el campo de la educación.

Finalmente, con relación a las preguntas de cierre planteadas, se logró identificar que a pesar de que parte de los estudiantes que participaron en el grupo de discusión mencionaron que existen aspectos que deben perfeccionarse para lograr una mejor experiencia de aprendizaje de la asignatura Cátedra Integradora; también están de acuerdo en que su inclusión en la malla curricular de la carrera de Profesionalización, ha resultado de gran importancia para su praxis profesional, por lo cual se sienten satisfechos al cursar la asignatura.

\section{Discusión}

La cátedra integradora como asignatura con base a lo expuesto a lo largo de los apartado anteriores, y particularmente con la información referencial, es posible identificarla como un área o aspecto en el cual es posible identificar a profundidad sobre los retos, aspectos específicos y objeto real en relación a las carreras de profesionalización que el ser humano cursa a lo largo de su vida universitaria, por lo que su importancia al día de hoy radica mucho más en la aplicación de esta dentro de las asignaturas para incentivar a resolver problemas o complementar los conocimientos del alumnado, antes de incurrir a dejar vacíos que comprometan el desarrollo profesional de estos (Aranda-Aranda, 2018).

Con base a lo expuesto en el apartado anterior, es posible a su vez argumentar que la cátedra integradora más allá de mostrarse como una asignatura, es la que permite identificar aspectos abordados desde perspectivas diversas para los profesionales que de una u otra manera buscan implantar mejoras en sus actividades o acciones puestas en marcha en el campo laboral, dado a que según lo que sustenta Durán et. al. (2018), la cátedra integradora tiene entre sus características permitirles a los estudiantes coordinar, armonizar, así como ir corroborando cada aspecto o tópico tratado en su proceso de aprendizaje, dado a que le otorga oportunidades a través de una serie de previos proyectos que se exigen llevar a cabo sobre temas asignados, para posteriormente diagnosticar a través de la revisión de estos, como se encuentra el estudiante.

Otra de las funciones que se le atribuye a la cátedra integradora, radica en los elementos de aportes que brinda en el desarrollo de determinados proyectos, dado a que inculca a como su nombre lo indica, a integrar saberes que ayuden a mejorar los perfiles de los estudiantes previo al ingreso a un tercer nivel de educación, esto en el caso de quiénes están en etapas previas de la universidad, como por ejemplo en los denominados preuniversitarios o propedéuticos, así como al termino de los semestres o asignaturas que cursan de una carrera universitaria, con lo cual, es posible 
además vincularlos con lo que podría ser el desarrollo de su proyecto final, o simplemente alimentar sus experticias de cara al desarrollo de proyectos que requieran un elevado nivel técnico-científico de información, análisis y presentación de resultados y en algunos casos de un propuesta de aplicación (Sánchez, 2018).

Entre los objetivos de la educación superior, se identificaron la generación de una formación lo más integral posible entre las personas que cursan sus carreras universitarias, esto por consiguiente hace referencia a buscar cultivar la aptitudes, competencias, así como complementar la experticia de las personas en muchos aspectos e inclusive de la personalidad, esto según lo contemplado por (Geycell, Herrera, y García, 2014), con lo cual logren tener un mejor desarrollo de su rama.

Dado a que la cátedra integradora busca que los estudiantes logren mantener aquellos conocimientos adquiridos en los primeros años de educación, y complementarlos con aquellas enseñanzas, tópicos o asignaturas que conllevan a la profesionalización, es importante que se tenga en consideración por parte de los docentes llevan adelante su práctica al impartirla, aplicar una metodología epistemológica que vaya acorde con el campo de actuación, identificando una base de información referencial que permita aplicar de manera idónea la malla o pensum educativo, para que así lograr que los estudiantes asimilen todo lo que se imparte dentro de las aulas de clases (Narváez-Peña, Salinas-Jumbo, y Curay-Jumbo, 2019).

Como se ha expuesto en párrafos anteriores, dentro de los procesos educativos el desarrollo de proyectos analíticos y con propuestas de solución son comunes, esto como parte del currículo que las carreras universitarias presentan, en la investigación formativa, se prepara a los estudiantes para que sean capaces de identificar problemas que se asocian a su profesión, con la ciencia, reconozcan tanto las líneas y sub líneas que la engloban, así como logren identificar la cátedra $\mathrm{y}$ proyectos integradores que se generan alrededor de estos, prácticas en relación a la comunidad, los trabajos o proyectos de grados y los ampliamente aplicados proyectos integrador de saberes (PIS).

A nivel del Ecuador, según lo establece a través del Reglamento de Régimen Académico, establecido a través del Consejo de Educación Superior (CES, 2017 ), particularmente dentro del Artículo 71, se instauró que dentro del proceso de educación de tercer nivel, que de acuerdo al grado o niveles educativos que cursan los estudiantes, deberá aplicarse e incentivarse un proceso investigativo acorde, con lo cual se asegurará los conocimientos y aptitudes de los alumnos basados en el completo entendimiento de los actuales escenarios de innovación científica, tecnológica, humanística y artística que son tan relevantes en la actualidad, donde a su vez, en el contexto formativo en el que se encuentran, la identificación y correcto manejo de metodologías epistemológicas e investigativas de alto rango, deberán verse reflejadas en los proyectos de tipo exploratorio así como descriptivos que se impongan aplicar (ArandaAranda, 2018).

Dentro del mismo reglamento del (CES, 2019) reformado, se acota particularmente en el Art. 38, que, dentro de las instituciones de educación superior, la función de investigación deberá abordarse considerando un nivel básico formativo, hasta lograr reflejar investigaciones de alto nivel científico; Se destaca dentro de este reglamento, que la interacción docente-estudiante, es obligatorio, impulsando una educación y aprendizaje 
mucho más activo por parte del estudiante, teniendo la libertad de despejar sus dudas sobre la asignatura o temas tratados dentro de la misma, aplicándose métodos innovadores como estrategia de enseñanza.

Por lo tanto, el estudiante universitario desde el inicio de su carrera, deberá empezar a desarrollar su sentido y capacidad de investigación, esto tomando en consideración que en su vida profesional como docente, tendrá que trabajar en el desarrollo de proyectos y tópicos que demanden de su completa experticia para tratarlos en las aulas, por lo cual, teniendo en consideración los retos y problemas que pudiesen surgir entre los alumnos para descifrar o comprender correctamente, el docente deberá saber transmitir lo que requerirá del proceso investigativo (Díaz-Valdés, Rodríguez-Guerra, y Valdés-Sáenz, 2017).

Si bien se identifica una limitada información en relación a lo que abarca la cátedra integradora, es posible finalmente definirla como una herramienta de apoyo entre los universitarios en su etapa de profesionalización, dado que esta permite que estos conozcan de manera real los retos de la asignatura, niveles y carreras que cursan, esto mediante previos proyectos investigativos y aplicación práctica, con lo cual lograran vincularse favorablemente con el contexto de su vida laboral y la sociedad que se busca beneficiar con la misma, por lo que radica ahí la importancia de esta herramienta y la aplicación de su estudio (Barros-Bastidas, Milanés-Gómez, y Rodríguez-Morales, 2019).

\section{CONCLUSIONES}

La cátedra integradora como herramienta en el proceso de profesionalización se la identifica fundamental en su aplicación y socialización, dado a que la misma permite que los estudiantes logren ampliar su perspectiva de lo que abarca tanto la carrera y asignaturas a las cuales se afrontaran desde los primeros niveles, hasta la etapa final de la universidad, y que será un recurso aplicado como parte de las estrategias que aplican a la metodología de enseñanza.

De los resultados que se lograron recabar del grupo de discusión en el que tuvieron participación tanto, los docentes como los estudiantes de la carrera de Profesionalización de la UNAE, fue posible determinar que la asignatura de cátedra integradora a pesar de presentarse como una herramienta que les ha permitido tener una mejor noción sobre lo que se requiere para tener un perfil profesional mucho más idóneo a los requerimientos actuales del sistema educativo de tercer nivel en el Ecuador, así como en el fortalecimiento de las habilidades para resolver problemas que se suscitan en relación a la carrera, de cara a los estudiantes, así como en relación a los entornos a los que se afrontan. Se identifica por ello, que la asignatura se ha implantado como una alternativa integral que favorece el desarrollo de quiénes están ya ejerciendo en la práctica docente y están en un proceso de profesionalización.

A su vez, los resultados permitieron determinar que actualmente la cátedra integradora como asignatura contribuye a que a través del análisis de escenarios tomando de base aspectos reales que surgirían en relación a la carrera, o bien en aspectos extracurriculares, que los estudiantes planteen alternativas para lograr dar solución a las situaciones que se presentan, las cuales no suelen ser constantemente favorecedores $\mathrm{o}$ positivas y por ende requieren de un tratamiento diferente para lograr un objetivo resolutivo, sin embargo, estas alternativas se planear a ser aplicadas desde los 
conocimientos propios de la práctica dentro del aula de clases.

De los resultados de las preguntas más específicas, fue posible identificar que si bien existe algo de satisfacción en el proceso y contenidos aplicados como parte de la cátedra, no estaría demás el que se apliquen contenidos, recursos, o estrategias que ayuden a hacer mucho más integral el aprendizaje, esto por ejemplo al momento de impartirse clases en relación al desarrollo de los proyectos que demanda la UNAE, los cuales deben cumplir con determinados requisitos científico-técnicos en la investigación y propuesta de mejora, solo de esta manera consideran los estudiantes que podrían entregar proyectos mucho más ajustados a lo requerido en el proceso de profesionalización de la universidad.

Si bien los docentes en el estudio de campo lograron estar de acuerdo con que las mejoras y ampliación de los contenidos para la asignatura de cátedra integrado son necesarios para el beneficio de los estudiantes, consideran que es una oportunidad para generar profesionales mucho más centrados a saber resolver de manera clara realidades contemporáneas del entorno en el que se desenvuelven, de otro modo se estaría simplemente aplicando una metodología de enseñanza pasiva, donde poca importancia se le da la participación activa que se espera de los estudiantes en proceso de formación para una profesión que demanda de un constante aprendizaje, apertura y compromiso para aprender a diario.

Otro de los aspectos destacables que el estudio permitió identificar, radica en el nivel de preparación de los docentes que se encuentran de cara a la formación de los estudiantes que siguen la carrera de docencia, dado a que son los maestros quiénes además de una metodología y estrategias eficientes de estudio, deben con el ejemplo educar y dejar de lado que cualquier barrera interrumpa el proceso de impartición de los conocimientos, para esto, el compromiso de los docentes también gira en relación a su aprendizaje constante, esto tanto a nivel académico, como en relación al entorno al que se afrontan y al que los estudiantes como profesionales se expondrán, buscando un equilibrio entre la flexibilidad, pero incitar a cumplir con las responsabilidades que una profesión como la docencia demanda.

La UNAE en el Ecuador ha logrado con el paso del tiempo convertirse en un referente oportuno en el proceso de formación de profesionales en diversos ámbitos, sin embargo, y teniendo claro el contexto del presente artículo, a nivel de la formación docente, es evidente que para lograr una educación integral, se requieren de aspectos que permitan promover el desarrollo de conocimientos y habilidades más exactas y específicas con relación a la realidad social y educativa en la que deberán desenvolverse los estudiantes de la carrera de Profesionalización, con lo cual se otorgará el mérito que requiere un área que en algunos escenarios es cuestionable en cuanto de calidad, por lo que es necesario que los docentes, el sistema educativo, y las universidades sean mucho más exigentes, con las metodologías aplicadas, para que los estudiantes se comprometan y no se estanque ante un sistema que se ve visualiza como poco eficiente.

Si bien los estudiantes en la investigación logran expresar que tienen perspectivas positivas de lo aprendido en la asignatura, dado a que a través de los proyectos que han realizado como parte del proceso de aprendizaje, cada vez están más familiarizados de lo que es importante considerar y abarcar 
en la profesión docente que buscarán consecuentemente generar entre los estudiantes con los que tengan contacto dentro de las aulas.

En relación a las preguntas finales que respondieron los estudiantes y docentes, se logró determinar que si bien la asignatura de cátedra integradora se presenta como herramienta de importante ayuda en el proceso de profesionalización, en la práctica como docentes ejerciendo, ha permitido tener mayor noción sobre cómo manejar determinadas situaciones, plantear proyectos, así como impulsar la investigación constante entre los estudiantes que tienen a su cargo, siendo provechoso dado a que se genera una aprendizaje de doble vía, atribuido al interés que los alumnos muestran en las aulas, lo que demanda de mayor compromiso por el profesional docente.

A pesar de las limitadas referencias de la asignatura de cátedra integradora, se ha logrado identificar la importancia de la misma, dado a que permite una socialización del estudiante sobre los posibles aspectos favorables y no tan favorables que en su vida profesional percibirán, para lo cual, deberán aplicar herramientas $y$ estrategias para afrontarlas $\mathrm{y}$ transmitir a su vez sus conocimientos a los estudiantes a los cuales instruyan.

\section{REFERENCIAS}

Aguilar, J., Apolo, D., y Rodríguez, M. (2018). El Buen vivir como desafío en la formación de maestros: aproximaciones desde la Universidad Nacional de Educación del Ecuador. Scielo, 23(77)

Aranda-Aranda, A. (2018). La investigación formativa $y$ el emprendimiento $e$ innovación en el currículo de carreras universitarias. Quito: Universidad Tecnológica Indoamérica-Ecuador.
Baptista, P., Hernández, R., y Fernández, C. (2014). Metodología de la investigación. México D.F.: McGRAW-HILL.

Barros-Bastidas, A., Milanés-Gómez, R., y Rodríguez-Morales, A. (2019). Profesionalización docente y formación desde un nuevo currículo en la Universidad de Guayaquil. Guayaquil: Scielo.

CES. (2017). Reglamento de Régimen Académico. Quito: CES.

CES. (2019). Regla-mento de Régimen Académico. Reformado 2019. Quito: CES.

Díaz-Valdés, K., Rodríguez-Guerra, Y., \& ValdésSáenz, M. (2017). La cátedra integradora en las mallas curriculares de las carreras universitarias. Revista Científica de FAREM-Estelí, 6(23), 97-107.

Durán, J., Rodríguez, F., y Montaluisa, A. (2018). Construcción de la propuesta educativa de la Facultad de Filosofía, Letras y Ciencias de la Educación de la Universidad Central del Ecuador. Quito: Universidad Central del Ecuador.

Geycell, E., Herrera, J., y García, Y. (2014). La integración de las influencias educativas de la universidad y la familia en la formación del profesional de la educación superior. La Habana: Universidad de Sancti Spíritus José Martí Pérez. .

Gobernación de Cañar. (12 de Julio de 2019). UNAE invita a obtener titulación de docentes en ejercicio a través de la postulación en la carrera de profesionalización. Obtenido de https://gobernacioncanar.gob.ec/unaeinvita-a-obtener-titulacion-de-docentesen-ejercicio-a-traves-de-la-postulacion-enla-carrera-de-profesionalizacion/

Moscoso-Zamora, V., y Quiñonez-Alvarado, E. (2018). Proyecto integrador de saberes, evidencia del resultado de aprendizaje. Revista Innova Research Journal, 3(3), 8494.

Narváez-Peña, M., Salinas-Jumbo, P., y CurayJumbo, I. (2019). Cátedra integradora una opción para incrementar los niveles de retención y titulación en las instituciones educativas. Espíritu Emprendedor TES, $3(3), 16-24$ 
Sánchez, A. F. (2018). Proyecto Integrador de Saberes de la Carrera de Pedagogía de las Ciencias Experimentales, Química y Biología de la Facultad de Filosofía, Letras y Ciencias de la Educación de la Universidad Central del Ecuador. Quito:
Universidad Central del Ecuador.

UNAE. (12 de Marzo de 2020). UNAE: Profesionalización. Obtenido de https://unae.edu.ec/pregrado/profesionali zacion/ 